\title{
Aplicación del Láser Escáner Terrestre (TLS) a la modelización de estructuras: precisión, exactitud y diseño de la adquisición de datos en casos reales
}

\author{
Terrestrial Laser Scanner application to structure modeling: precision, \\ accuracy and design of data acquisition in real case studies

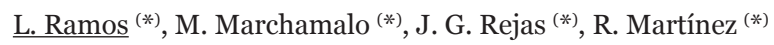

\section{RESUMEN}

El láser escáner terrestre se emplea en numerosas aplicaciones de Ingeniería, debido a las prestaciones de los equipos y a las precisiones alcanzadas. Se presenta la aplicación del TLS a la modelización de estructuras y paramentos verticales. Se analizan los factores que influyen en la precisión final: registro de nubes, tipo de dianas y el efecto del ángulo y distancia de escaneo. Se realizó una prueba de campo con testigos calibrados, obteniendo valores reales de precisión y exactitud para los rangos de distancia más usuales en Ingeniería.

Se propone y valida el empleo de gráficos patrón que relacionan las variables precisión y exactitud con los factores distancia y ángulo de escaneo para el diseño de trabajos de campo. Se expone su aplicación al diseño de los escaneos para el control de movimientos de una presa. Se realizan recomendaciones para la aplicación de la técnica TLS a grandes estructuras.

Palabras clave: Láser escáner terrestre; control de movimientos; precisión; grandes estructuras; presas.

\section{ABSTRACT}

Terrestrial laser scanner is employed in a wide range of engineering applications, because of the specifications of TLS units and the good precision reached. This paper presents the application of TLS to structures and walls modeling. It analyzes the factors that influence final accuracy: cloud registration, target type and the effect of scanning angle and distance. A field test was performed with calibrated panels, obtaining real precision and accuracy values for the most common distance ranges in engineering.

We propose and validate the usage of pattern graphs relating precision and accuracy versus distance and scanning angle for field survey design. The application of pattern graph in the design of field scan surveys for dam movement control is also presented. Recommendations are made for the application of TLS technique to large structures.

Keywords: Terrestrial laser scanner; movement control; precision; large structures; dams.

ETSI de Caminos, Canales y Puertos - Universidad Politécnica de Madrid (España).

Persona de contacto/Corresponding author: luis.ramos.alcazar@upm.es (L. Ramos)

Cómo citar este artículo/Citation: Ramos, L., Marchamalo, M., Rejas, J. G., Martínez, R. (2015). Aplicación del Láser Escáner Terrestre (TLS) a la modelización de estructuras: precisión, exactitud y diseño de la adquisición de datos en casos reales. Informes de la Construcción, 67(538): e074, doi: http://dx.doi.org/10.3989/ic.13.103.

Licencia / License: Salvo indicación contraria, todos los contenidos de la edición electrónica de Informes de la Construcción se distribuyen bajo una licencia de uso y distribución Creative Commons Reconocimiento no Comercial 3.o. España (cc-by-nc). 


\section{INTRODUCCIÓN}

El láser escáner terrestre es ya una tecnología aceptada en un amplio campo de aplicaciones, entre las que podemos señalar: arqueología, estudios arquitectónicos, modelos as-built, instalaciones industriales, inventario y GIS, seguimiento del comportamiento de estructuras y elementos estructurales, movimientos del terreno, minería y canteras, túneles y galerías, atestados, etc.

Esta versatilidad se debe a la variedad de prestaciones de los equipos TLS. Hay tres principios de medida electro-ópticos para determinar distancias entre un emisor y un objeto: tiempo de vuelo, triangulación e interferometría. Los TLS utilizados en Arquitectura e Ingeniería se basan en el principio del tiempo de vuelo y se diferencian según el método de medida de éste en: directos o pulsados (señal emitida por pulsos) e indirectos o por fase (señal continua con amplitud modulada). El método indirecto o de fase es el más exacto de los dos; consigue una gran concentración de energía pero el rango de distancia apto para medir es más limitado (hasta los 100m). El método directo o pulsado se usa para medir distancias mayores, hasta 300 metros.

$\mathrm{Al}$ ser el TLS un sistema de medida sin prisma, que resuelve la posición en función de la intensidad de la señal reflejada, es muy dependiente de las propiedades físicas del rayo, en particular su coherencia espacial, de la distancia y del ángulo de incidencia del rayo láser respecto del objeto observado y por último de las propiedades reflectoras de la superficie del objeto, color y textura; sin olvidar los factores ambientales.

Las especificaciones que se dan de cada equipo TLS suelen ser insuficientes cuando, como suele acontecer, se necesita extrapolar dichas características a distancias de trabajo mayores. En las especificaciones del fabricante se proporciona el rango de distancias del aparato, pero usualmente, los valores de la precisión y la exactitud se indican sólo para una determinada distancia. Como a mayor distancia se produce una mayor dispersión del rayo, y por tanto menor intensidad de retorno, se plantea la necesidad de conocer hasta qué distancia se pueden asumir los valores de precisión y exactitud dados en las especificaciones y qué valores se presentan a distancias mayores. Estos datos son fundamentales para la planificación de trabajos de escaneo en múltiples aplicaciones de ingeniería y arquitectura, que requieren la obtención de modelos digitales 3D de paramentos, fachadas, revestimientos o detalle constructivos.

Estas restricciones, unidas a las limitaciones por visibilidad, obligan en muchos casos a plantear escaneos parciales desde varios puntos y posteriormente realizar el registro de ellos para unificarlos en un mismo sistema de coordenadas. El registro de nubes requiere emplear dianas para materializar puntos de control. Cada fabricante tiene sus propias dianas y el software de cada TLS está ya programado para reconocerlas. Las dianas suelen ser planas o esféricas, por lo que es necesario evaluar la idoneidad de cada uno de estos tipos en trabajos concretos.

Por otro lado hay que conocer la naturaleza de la nube de puntos $3 \mathrm{D}$, que es el producto que se obtiene con el TLS. El carácter de nube no sólo se debe a la gran cantidad de puntos que se obtienen sino también porque estos puntos forman una nebulosa que envuelve al objeto observado. Y se puede decir que no hay dos nubes de puntos iguales de una misma superficie, tomadas con el mismo aparato desde la misma posición. Esto implica que no se debe tratar la nube de puntos con análisis punto a punto como en la topografía clásica sino con análisis de superficies. Este hecho es ineludible en estudios de comportamiento de un paramento a lo largo del tiempo. Por ello es necesario un post-proceso en oficina de las nubes de puntos para la generación de las correspondientes mallas, normalmente de triángulos.

En numerosas publicaciones sobre los equipos TLS se presentan resultados sobre la influencia en la intensidad de la señal recibida de la distancia de escaneo, el ángulo de incidencia (horizontal y vertical) y la reflectancia, por colores y texturas. Normalmente se presentan mediante familias de curvas donde se muestran la variación de la intensidad respecto a un factor manteniendo constante en cada curva un segundo factor (1) (2). La literatura presenta algún ejemplo de representación tridimensional (2). También hay estudios de la variación de la exactitud con la distancia y el ángulo de incidencia, pero estudiados cada uno de estos factores por separado (3).

La caracterización de la influencia de la distancia de escaneo y ángulo de incidencia en la precisión y exactitud finales debe hacerse conjuntamente debido a la naturaleza cónica de la emisión laser. Para ello se propone un formato de gráficos zonales, en un espacio distancia/ángulo de incidencia, que representa mediante isolíneas las relaciones entre ambos parámetros que proporcionen iguales niveles de precisión y exactitud. Estos gráficos zonales serán de gran utilidad para la selección del equipo TLS adecuado y la planificación del trabajo de campo. En este trabajo se propone un modelo de gráfico patrón, para relacionar la precisión y la exactitud con dos factores fundamentales para el diseño del escaneo: distancia y ángulo de incidencia del rayo. Este gráfico será específico para cada equipo TLS y servirá para la correcta elección y formulación del equipo, red de estaciones y plan de trabajo.

El presente trabajo tiene como objetivo principal proporcionar a los arquitectos e ingenieros información validada sobre los parámetros que influyen en la precisión y exactitud de los equipos TLS y herramientas para el diseño de trabajos de campo y la explotación de los resultados. Con este fin se realizaron pruebas experimentales en campo para responder a los siguientes objetivos específicos: a) determinar la influencia del tipo de diana en la precisión; b) evaluar la precisión y exactitud en la determinación de diferencias en un paramento y c) validar los gráficos patrón dispersión/precisión y exactitud para la explotación de resultados. Se particularizan los resultados para su aplicación en el control de los movimientos estacionales de las presas. Esta tarea ha sido abordada por otros autores (4) (5), en un plano más general.

\section{METODOLOGÍA}

\subsection{Prueba de campo}

Con el fin de completar los objetivos de la investigación se realizó una prueba de campo con testigos calibrados, obteniendo valores reales de precisión y exactitud para las distancias más usuales en Ingeniería. En el mismo se analizaron los factores que influyen en la precisión final: método de registro de nubes, empleo de discos o esferas y el efecto del ángulo y distancia de escaneo. 
La metodología se validó con un equipo TLS concreto y se aplicó al control de los movimientos de una presa. Esto requiere trabajar con valores de precisión por debajo de los 5 $\mathrm{mm}$ para distancias que superen los $100 \mathrm{~m}$, debido a la magnitud de estas estructuras (6). Por ello se seleccionó un equipo entre los que utilizan el principio de tiempo de vuelo con medición directa o por pulsos, el ScanStation C10 de Leica. De acuerdo con las especificaciones dadas por el fabricante (7), en la Tabla 1 se recogen algunas de las características de este TLS.

La fase experimental se realizó en el parking de la Escuela Técnica Superior de Ingenieros de Caminos, Canales y Puertos de la Universidad Politécnica de Madrid (ETSICCP). Se seleccionó esta ubicación debido a que se podía trabajar a distancias superiores a los $100 \mathrm{~m}$ y a que la fachada principal de la Escuela presenta un acabado en hormigón visto. Este acabado es similar al de las presas, caso al que se aplican los resultados en la discusión de este estudio. En este parking, el Laboratorio de Topografía de la ETSICCP tiene una red topográfica con coordenadas, materializada con clavos. (Figura 1).

El propósito fue doble:

- Por un lado determinar la incertidumbre y precisión que se obtienen empleando dianas esféricas y de disco con la Leica ScanStation C10 a distancias medias con el fin de utilizarlas para el registro de las nubes de puntos.

- En segundo lugar, establecimiento mediante escaneos sucesivos del umbral de detección de pequeños movimientos simulados en el paramento de la fachada mediante testigos de espesor calibrado.

Además del Leica ScanStation C10, se emplearon dos dianas semiesféricas, que integran disco y esfera. $\mathrm{Al}$ no disponer de vértices en la zona, tanto la C10 como las dianas se estacionaron sobre trípodes con bases nivelantes. El centrado del C10 se realiza con plomada láser y el de las dianas con burbuja y plomada óptica. Las dianas se colocaron en los puntos A3 y B9 y se realizaron los escaneos desde los puntos B4 y B5, resaltados en la Figura 1. La diana semiesférica era idónea para esta prueba puesto que no era necesario repetir el centrado bastando solo con girar la diana.

Uno de los objetivos de la prueba era determinar las precisiones obtenidas en las medidas de dianas esféricas y de disco estando éstas en la misma posición. Como se ha dicho, la diana semiesférica era idónea y se tuvo en cuenta esta ventaja a la hora de establecer el orden de los escaneos. El escaneo de las dianas se realizó con la máxima densidad de puntos que puede dar el aparato. En el caso del Leica C10 es de $1 \mathrm{~mm}$ para el rango de distancias del ensayo. El diámetro de las esferas era de $15 \mathrm{~cm}$. Según las especificaciones del fabricante, la incertidumbre o precisión del $\mathrm{C} 10$ en la «adquisición del objetivo» (la diana) a $50 \mathrm{~m}$ de distancia es de $2 \mathrm{~mm}$ ( 1 sigma = desviación típica).

La simulación del movimiento del paramento vertical se hizo colocando unos testigos sujetos en el paramento de la fachada del edificio. Los testigos consistían en tacos consolidados y calibrados de papel de $80 \mathrm{gr} / \mathrm{cm}^{2}$. Se colocaron en dos zonas: una más próxima y casi perpendicular a la posición del láser escáner (zona 1) y otra más alejada y oblicua (zona 2). En la zona 1 se colocaron tres testigos con espesores de $1,5 \mathrm{~cm}, 0,8 \mathrm{~cm}$ y 0,6 $\mathrm{cm}$ y en la zona 2 se colocaron tres testigos con espesores de $3,5 \mathrm{~cm}, 1,0 \mathrm{~cm}$ y $0,5 \mathrm{~cm}$. En la Figura 1 se presentan las visuales desde las estaciones y las distancias de escaneo anotadas. El ángulo de incidencia vertical de las visuales a los testigos era del orden de 94 grados centesimales. El orden de los escaneos realizados (dianas y fachada) aparecen en la Tabla 2.

Tabla 1. Características del TLS ScanStation C10 de Leica.

\begin{tabular}{|l|l|l|c|}
\hline \multicolumn{4}{|c|}{ Leica ScanStation C1o } \\
\hline Principio de medida & Pulsado & Alcance $(\mathrm{m})$ & $0.1-300$ \\
\hline Color láser & Verde $(532 \mathrm{~nm})$ & Resolución angular (“) & $12 / 12$ \\
\hline Clase de láser & $3 \mathrm{R}$ & Precisión punto $(\mathrm{mm}-1 \mathrm{~s})$ & $6(1-50 \mathrm{~m})$ \\
\hline Campo de visión & $360 \times 270$ & Precisión Superf. (mm - 1s) & $2(1-50 \mathrm{Om})$ \\
\hline Velocidad & $50.000 \mathrm{pts} / \mathrm{s}$ & Precisión diana (mm - 1s) & $2(1-50 m)$ \\
\hline
\end{tabular}

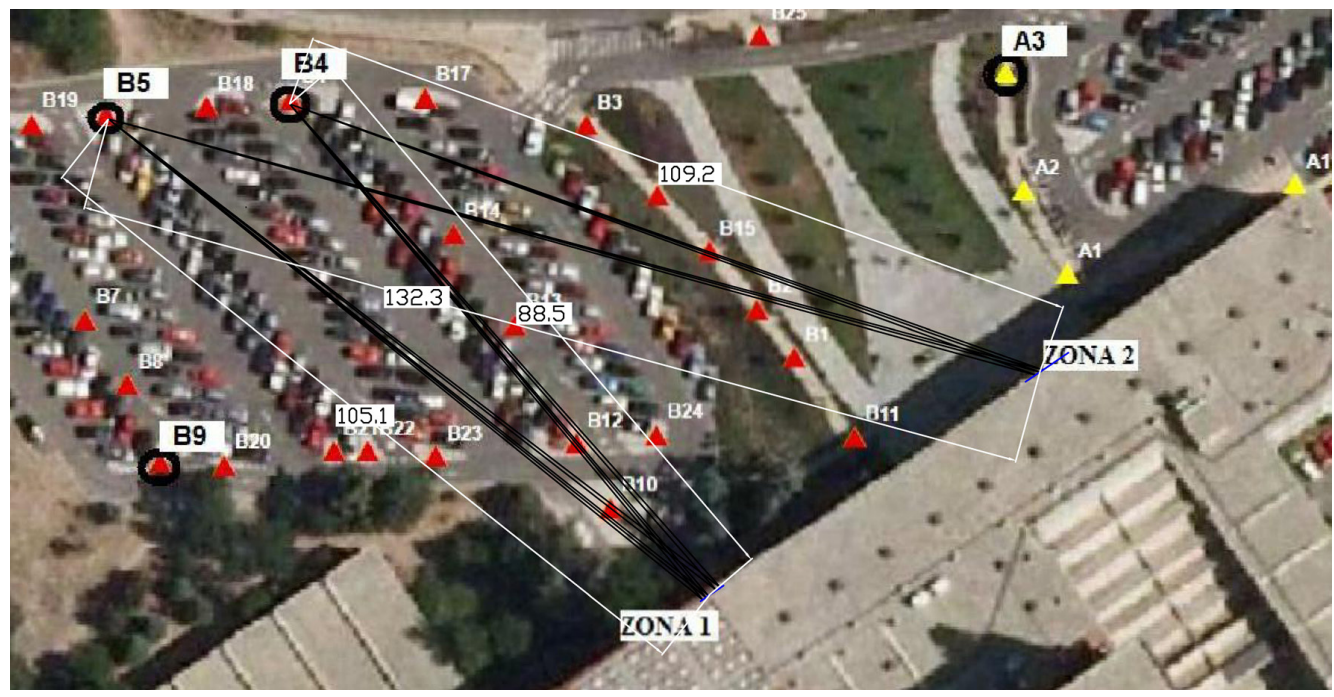

Figura 1. Lugar de la realización del ensayo. 


\subsection{Proceso de datos}

Se utilizó el programa Cyclone de Leica para el tratamiento de las nubes de puntos de las dianas y para el registro de las nubes de puntos de la fachada. Se hizo el registro de las nubes de puntos tomadas desde B5 para transformarlos al sistema de coordenadas local de B4. El análisis posterior de las nubes de puntos se realizó con el programa 3DReshaper. Para ello se crearon las respectivas mallas de triángulos de las nubes de puntos exportadas desde Cyclone y posteriormente se compararon las mallas de la fachada dos a dos para detectar las diferencias entre escaneos.

Los valores de precisión y exactitud se calcularon como diferencia entre dos superficies, que simulan un pequeño movimiento del paramento. La incertidumbre o precisión se calculó como el valor $1 \sigma$ de las diferencias entre las mallas con y sin testigos. Una incertidumbre baja significa una precisión alta. La exactitud corresponde a la media de las desviaciones de las diferencias, en valor absoluto, entre las mallas con y sin testigo respecto al espesor real del testigo.

Los pasos seguidos han sido:

1. Identificar las nubes de puntos correspondientes a los escaneos realizados.

2. Realizar el mallado de dichas nubes de puntos.

3. Comparar (medir diferencias) entre mallas de cada zona, con y sin testigos. Esto genera nuevas mallas con un valor adicional en cada vértice de éstas: la diferencia entre las dos mallas de referencia. La malla de las diferencias se obtiene lanzando normales desde los vértices de una malla a la otra y midiendo las distancias. Este valor de la diferencia se añade como una cuarta dimensión a los vértices de la malla de las diferencias.

4. Aislar cada una de las zona de cada testigo en las mallas de las diferencias generando las correspondientes sub-mallas

Tabla 2. Escaneos realizados a dianas y paramento vertical.

\begin{tabular}{|c|c|c|}
\hline ESTACIÓN & OBJETIVO & OBS. \\
\hline \multirow{7}{*}{ B4 } & ESFERA en A3 & \\
\hline & ESFERA en B9 & \\
\hline & ZONA 1 & Sin testigos \\
\hline & ZONA 2 & Sin testigos \\
\hline & ZONA 1 & Con testigos \\
\hline & DISCO en A3 & \\
\hline & DISCO en B9 & \\
\hline \multirow{7}{*}{ B4 BIS } & DISCO en A3 & \\
\hline & DISCO en B9 & \\
\hline & ZONA 2 & Sin testigos \\
\hline & ZONA 1 & Con testigos \\
\hline & ZONA 2 & Con testigos \\
\hline & ESFERA en A3 & \\
\hline & ESFERA en B9 & \\
\hline \multirow{8}{*}{$\mathrm{B}_{5}$} & ZONA 1 & Con testigos \\
\hline & ZONA 2 & Con testigos \\
\hline & ESFERA en A3 & \\
\hline & ESFERA en B9 & \\
\hline & ZONA 1 & Sin testigos \\
\hline & ZONA 2 & Sin testigos \\
\hline & DISCO en A3 & \\
\hline & DISCO en B9 & \\
\hline
\end{tabular}

para el tratamiento de la información de los vértices (coordenadas $\mathrm{x}, \mathrm{y}, \mathrm{z}$ y diferencia) en una hoja de cálculo.

5. Determinar la incertidumbre/precisión y la exactitud en cada sub-malla y obtener la media de los valores correspondientes a los testigos de la zona 1 y de la zona 2.

\section{RESULTADOS}

\subsection{Precisión en función del tipo de diana}

Los resultados obtenidos tras el calibrado de esferas y discos con el programa Cyclone confirman las especificaciones del aparato dando resultados por debajo de los $2 \mathrm{~mm}$, en el caso de las esferas hasta los $120 \mathrm{~m}$. Los resultados se recogen en la Tabla 3 y en la Figura 2.

Los procedimientos que utiliza Cyclone para determinar el centro de la diana son distintos tratándose de un disco o de una esfera. Cada TLS está configurado para trabajar con unas dianas determinadas que diseña el fabricante del equipo. Al efectuar el escaneo de las dianas, se introduce a priori en el programa del TLS qué tipo de diana va a escanear y su tamaño. Con ello el TLS se configura para generar una nube con la máxima densidad de puntos.

Las dianas de disco utilizan sectores o coronas de diferente color con lo que las reflectividades serán diferentes y, por tanto, las intensidades recibidas por el aparato. A partir de ahí se determina el centro del disco. No hay que olvidar que el tamaño del disco es un dato que conoce el programa igual que el tamaño de la esfera. Las dianas esféricas suelen ser blancas, no hay variaciones de la intensidad debido a la reflectividad, pero en cambio es un objeto tridimensional por lo que se utiliza esta característica geométrica para determinar la posición del centro.

En la Tabla 3 y Figura 2 se presentan los resultados de incertidumbre en la determinación del centro de dianas de disco y esféricas para distintas distancias de escaneo. Se constata que, en el caso del C10, para distancias superiores a los 60 metros deberían utilizarse dianas esféricas.

En la Figura 3 se representan las nubes de puntos de los escaneos de disco y esfera con el ajuste realizado por Cyclone para determinar la posición de los centros. A la derecha se muestra la superposición de ambas dianas y la medida de la distancia desde el centro del disco al centro de la esfera.

La utilización de dianas se encuadra en escenarios de trabajo en los que, por tamaño de la estructura o razones de visibilidad, sea necesario realizar varias puestas en estación del TLS para obtener un escaneo con precisión suficiente. Habrá que estudiar el entorno del objeto a estudio a fin de determinar la ubicación idónea para la(s) puesta(s) en estación del TLS, con un criterio de máxima economía de medios y minimizar el tiempo de trabajo en campo, por lo que:

- Se debería contar con la existencia de una red local georreferenciada utilizable por las estaciones totales y también por los TLS.

- Se debe estudiar si es factible hacer todo el escaneo del objeto desde un solo vértice evitando el posterior registro de las nubes de puntos. Para ello se analizan las diferentes distancias y ángulos de barrido del TLS desde los posibles vértices al objeto de estudio. 


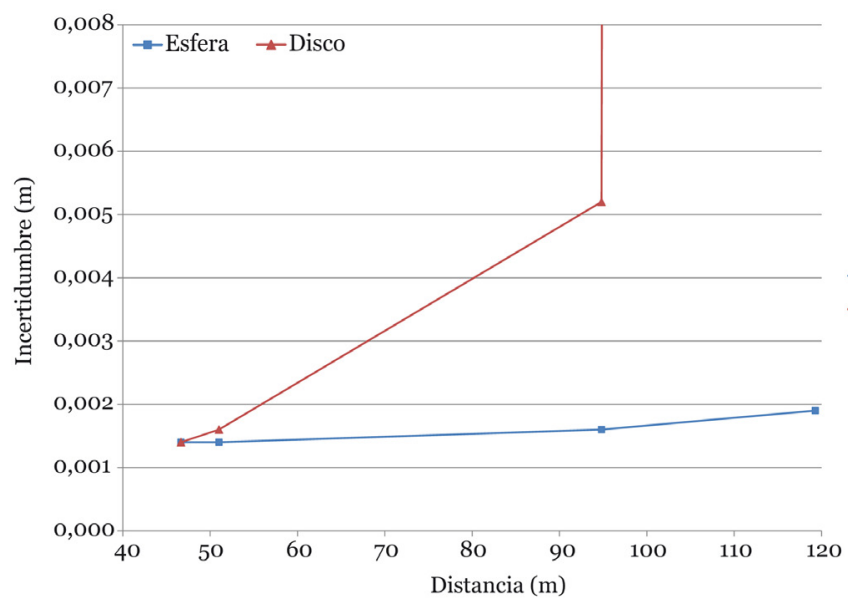

Figura 2. Resultados de las observaciones a las dianas.

- En el caso de tener que hacer escaneos desde varias posiciones es necesario estudiar el número y ubicación de dianas para determinar qué tipo de diana utilizar.

- Hay que tener en cuenta la facilidad de acceso a los puntos donde se vayan a colocar las dianas. Es desaconsejable contar con dianas en el paramento del objeto a escanear por dificultad en su instalación y conservación, además que al no ser posiciones fijas obliga a instalar mayor número de éstas. Por ello, es recomendable colocar las dianas en puntos fijos, con ello se necesitarán menos dianas.

\subsection{Precisión y exactitud en la determinación de diferencias en un paramento}

El segundo objetivo de la prueba realizada era la detección mediante escaneos sucesivos de pequeños movimientos simulados en un paramento vertical y obtener la precisión y exactitud de los movimientos detectados en función de la distancia y ángulo de incidencia horizontal desde donde se realizaron los escaneos. Con los datos obtenidos se crea el gráfico patrón que determina el espacio distancia-ángulo de incidencia abarcable desde cada estación y poder así determinar la estación(es) idónea(s) para el TLS.

En la Figura 4 se muestra una de las mallas obtenidas al final del paso 3. En concreto, corresponde a la comparación de los escaneos de la zona 2 con testigos y sin testigos, realizados desde las posiciones $\mathrm{B} 4$ bis y $\mathrm{B} 4$ respectivamente. La distancia de escaneo era alrededor de los $100 \mathrm{~m}$ y el ángulo de incidencia horizontal alrededor de los 59 grados centesimales. En el gráfico se utiliza una gradación de colores para representar las diferencias entre las mallas comparadas. Se observa que en la zona de fachada sin testigos las diferencias están por debajo de $3 \mathrm{~mm}$ (color verde) y que las zonas de los tres testigos presentan diferencias positivas y colores acordes. También se muestran en el gráfico etiquetas de valores concretos de las diferencias en varios puntos de la malla. En la Figura 5 se muestran las secciones por un plano horizontal medio de las dos mallas comparadas de la zona 2 con los testigos de espesores $3,5 \mathrm{~cm}, 1,0 \mathrm{~cm}$ y 0,5 $\mathrm{cm}$ respectivamente. La primera conclusión es que las imágenes de las Figuras 4 y 5 tienen una exactitud aceptable; los resultados son fiables.

Es ilustrativo mostrar superpuestas la nube de puntos proveniente del escaneo (datos originales), la malla generada por regresión de la nube de puntos (datos de proceso) y la sección horizontal tomada de la malla (datos de verificación) (Figura 6).

Si hubiera que explicar la diferencia entre la topografía tradicional punto a punto y las nuevas técnicas de captación de superficies basado en las nubes de punto $3 \mathrm{D}$, se podría ilustrar con imágenes como las de la Figura 6. En esta figura se han incluido una vista en planta, un alzado frontal y una vista lateral de la nube de puntos (en rojo), la malla de triángulos (en verde) y una sección horizontal (en negro), correspondiente al área de la fachada donde posteriormente se colocó el testigo de espesor $3,5 \mathrm{~cm}$ en la zona 2. La distancia entre

Tabla 3. Resultados de las observaciones a las dianas.

\begin{tabular}{|l|c|c|c|}
\hline \multicolumn{1}{|c|}{ Medida } & $\begin{array}{c}\text { Distancia } \\
\text { (m) }\end{array}$ & $\begin{array}{c}\text { Incertidumbre (m) } \\
\text { Esfera }\end{array}$ & $\begin{array}{c}\text { Incertidumbre (m)* } \\
\text { Disco }\end{array}$ \\
\hline B4-A3 & 94,808 & 0,0016 & 0,0052 \\
\hline B4 bis-A3 & & 0,0016 & 0,0068 \\
\hline B4-B9 & 51,012 & 0,0014 & 0,0016 \\
\hline B4 bis-B9 & & 0,0014 & o,o019 \\
\hline B5-A3 & 119,288 & 0,0019 & n.d. \\
\hline B5-B9 & 46,662 & 0,0014 & o,o014 \\
\hline
\end{tabular}

*Distancia del centro del disco al centro de la esfera.

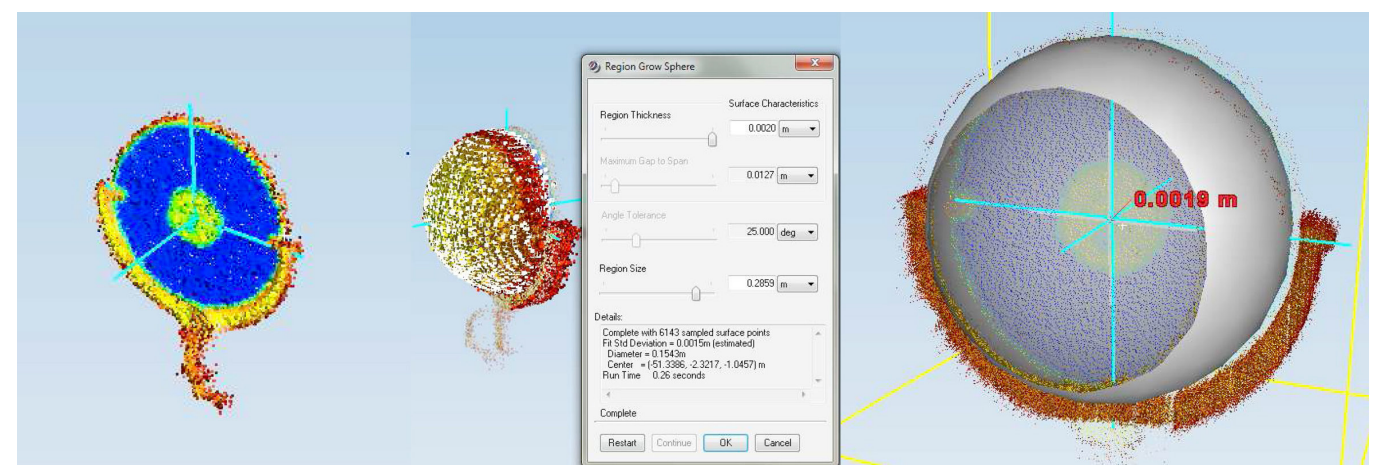

Figura 3. Nubes de puntos de disco y esfera en Cyclone. 


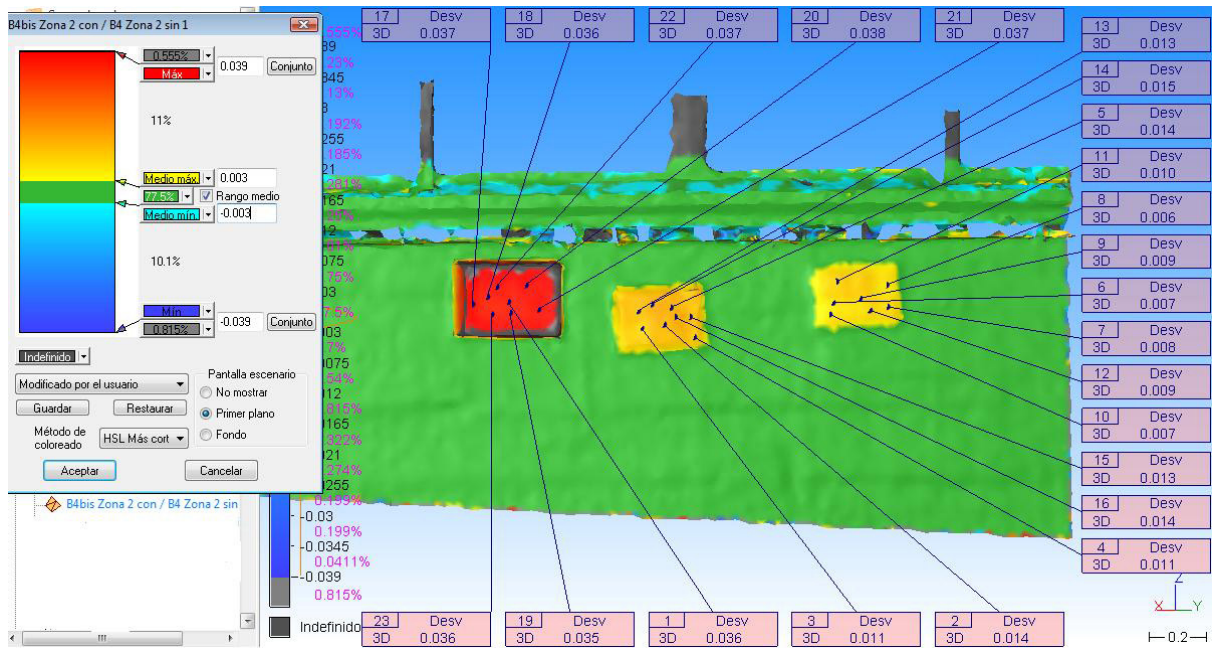

Figura 4. Comparativa entre mallas realizada con 3DReshaper.

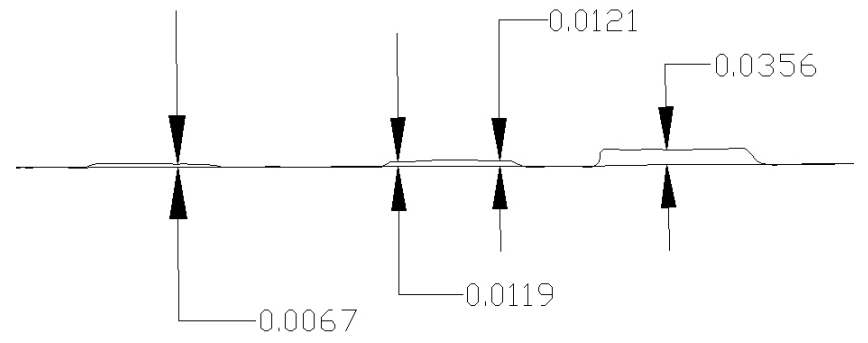

Figura 5. Secciones de las mallas del paramento por un plano horizontal.

rayos de la nube de puntos es de $6 \mathrm{~mm}$ y el lado promedio de los triángulos de la malla de $2,5 \mathrm{~cm}$.

En la Figura 4 se representó esa cuarta dimensión (la diferencia entre dos mallas) obtenida en el paso 3 del procedimiento referido arriba. Habría que preguntarse si los resultados en relación a la exactitud de cada una de las mallas por separado, comparadas con el paramento real dan los mismos niveles de aproximación que con las diferencias. Esto queda para posteriores pruebas, si bien habría que comentar que se trataría de aplicaciones diferentes de esta tecnología, resaltando que lo que se pretendía en este test era comprobar la idoneidad de la tecnología del TLS para estudios de pequeños movimientos de paramentos verticales.

\section{LOS GRÁFICOS PATRÓN DISPERSIÓN/ PRECISIÓN Y EXACTITUD DE UN EQUIPO TLS PARA EL CONTROL DE MOVIMIENTOS PEQUEÑOS}

En este trabajo se presenta un prototipo de lo que sería el gráfico patrón dispersión/precisión en función de la distancia y el ángulo de incidencia (en este caso el horizontal o acimutal, pero que sirve también para el ángulo vertical) que se propone como herramienta idónea a la hora de planificar un trabajo con TLS (Figura 7). Con los escaneos realizados durante la prueba se obtuvieron cuatro pares de valores distancia/ángulo de incidencia (Figura 1), que representan valores promedio de las desviaciones típicas de los resultados obtenidos en los testigos de cada zona. El rango que cubren es significativo y los resultados confirman los niveles de incertidumbre obtenidos en las dianas.

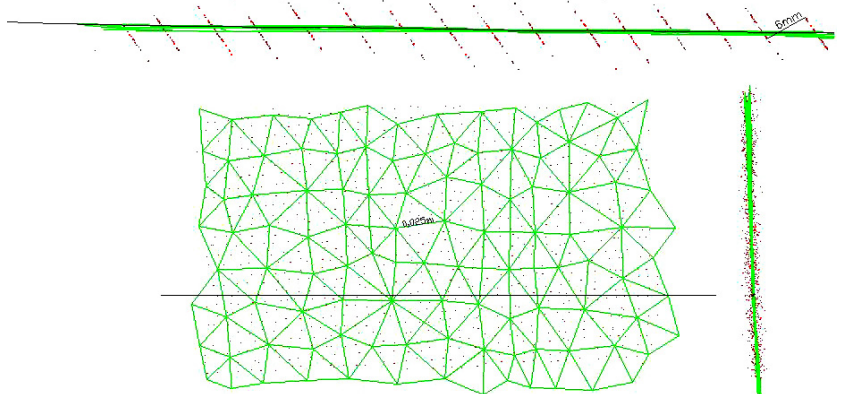

Figura 6. Vista en planta, alzado y lateral de la nube de puntos, la malla de triángulos y una sección horizontal.

Dispersión y precisión se refieren a la misma aptitud del aparato: la capacidad de repetir los mismos valores en mediciones sucesivas realizadas en las mismas condiciones; pero son contrapuestos, a mayor dispersión menor precisión. Sin embargo, se puede plantear una forma de representación que refuerza y complementa ambas formas de presentar la misma medida con el uso de símbolos proporcionales y escala de grises. A fin de no contravenir los principios de las propiedades perceptivas de las variables visuales de los símbolos se insiste en denominar a esta representación gráfico dispersión/precisión, con los dos nombres, queriendo decir que se utiliza una técnica para referirse como dispersión (símbolos proporcionales) y otra técnica como precisión (escala de grises). El resultado parece muy adecuado para tratar datos de láser escáner en cuanto la mayor dispersión se representa con un círculo mayor y la mayor precisión con el color negro. De este modo un punto pequeño y negro representa el dato de mejor calidad y un círculo grande y gris claro representa el dato de menos calidad (Figura 7). Este gráfico es parcial, ya que el origen en abscisas y ordenadas debería ser el valor cero y en las distancias cubrir todo el rango dado en las especificaciones del equipo.

Los valores de dispersión/precisión obtenidos siguen estando por debajo de los $2 \mathrm{~mm}$. Si hubiera más datos, para mayores intervalos de distancia y ángulos, todos estos valores se hubieran representado como de $2 \mathrm{~mm}$. No se ha hecho aquí para ejemplarizar la técnica de representación propuesta. El gráfico se completa con la correspondiente representación de isolíneas, con lo que obtendríamos una zonificación en el plano distancia/ángulo de incidencia que nos permitiría de- 


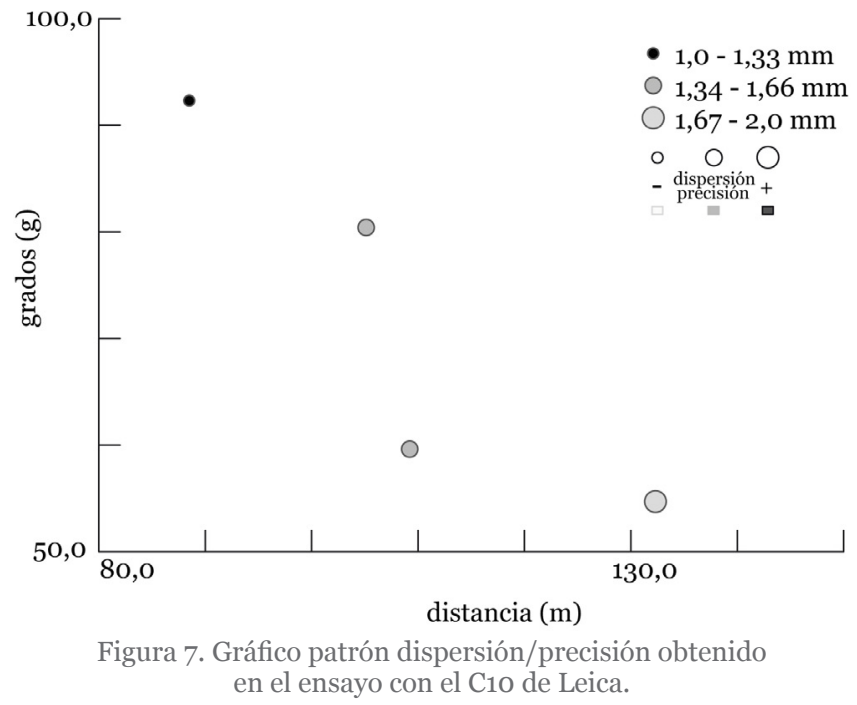

terminar las fronteras respecto al alcance de cada nube de puntos en función del propósito de cada trabajo.

En relación a la representación de un gráfico patrón de la exactitud del TLS no tenemos esa dualidad en el concepto. La exactitud, como proximidad del valor medido con el valor real, referido a las medidas de movimientos se representará mejor simplemente con un mapa de isolíneas. En la Tabla 4 se recogen los valores medios de la exactitud correspondiente a las sub-mallas de las diferencias.

Los datos presentados corresponden a la comparación de las mallas de la fachada con y sin testigos desde la misma estación. También se compararon entre sí las mallas generadas desde las estaciones B4/B4bis y B5. Para ello se hizo el registro de las nubes tomadas desde la estación B5 para pasarlas al sistema de coordenadas local de B4. Los valores obtenidos tanto en dispersión/precisión como en exactitud fueron iguales que los anteriores. Por último se compararon las mallas del paramento de la fachada sin testigos realizadas desde las dos estaciones dando valores de exactitud por debajo de los $2 \mathrm{~mm}$.

Habría que hacer un comentario respecto a la influencia de otros factores en el resultado: ángulo de incidencia vertical, color del objeto y textura. Se ha apuntado más arriba que el gráfico patrón incertidumbre/precisión en función de la distancia y el ángulo de incidencia horizontal sirve también para el ángulo de incidencia vertical. En efecto los mecanismos del TLS para el barrido en horizontal y vertical son iguales. Por ello sería mejor generar un gráfico patrón distancia-ángulo de incidencia espacial medido éste como el ángulo entre la dirección del rayo y la normal a la superficie en el punto de incidencia del rayo. La obtención del gráfico patrón distancia-ángulo de incidencia espacial queda pendiente en fases posteriores de esta línea de investigación. El color del objeto tiene influencia en la reflectividad y los estudios demuestran que para distancias grandes y objetos obscuros la precisión y exactitud decrecen (2). Respecto a la textura habría que considerar rangos de superficies duras y blandas, lisas y rugosas. Nuestra opinión es hacer planos bidimensionales distanciaángulo de incidencia para diferentes colores y texturas.

\subsection{Aplicación de los gráficos patrón dispersión/ precisión y exactitud de un TLS al control de movimientos de las presas en función de su geometría}

Uno de los campos de aplicación de la tecnología del láser escáner terrestre es el control estacional de los movimientos de una presa. En la monitorización de presas se utilizan instalaciones permanentes que dan registros continuos de los movimientos absolutos o relativos en ciertos puntos de la presa (8) y (9). La tecnología TLS proporciona el registro temporal de los movimientos de todo el paramento de la presa y esa es su gran aportación. Una de las ventajas del TLS frente a otras técnicas como el radar terrestre (10) es que, aunque este último puede ser más preciso que el TLS, requiere de una instalación más costosa y necesita mayor vigilancia. Sin embargo, el TLS aprovecha los vértices fijos de la red local de la presa que normalmente ya existe, tanto para la colocación del equipo como de las dianas.

La gran diversidad de situaciones que se pueden presentar en una presa en lo relativo a accesibilidad y visibilidad obligan a hacer un estudio detallado de cada caso y seguramente plantearse la conveniencia de colocar más vértices fijos para el máximo aprovechamiento de recursos y optimización de procedimientos involucrados en la realización de los escaneos. Con ello se puede minimizar los estacionamientos del TLS y, si fuera posible, realizar uno sólo con lo que se eliminaría la necesidad de tener que hacer el registro de las diferentes nubes de puntos en el post-proceso. Esto último va asociado con la necesidad del uso de dianas en mayor o menor número y la reducción del tiempo invertido en los escaneos.

A modo de ejemplo, en la Figura 8 se presentan dos configuraciones de cerrada de un embalse, una en arco de 150 metros de radio y otra de directriz recta. Se muestran las medidas geométricas de distancias y ángulos desde tres posiciones aguas abajo a los extremos y punto medio de ambas cerradas. A la vista del mapa patrón de la Figura 8 y de la Tabla 3 se puede planificar desde qué posiciones y con qué cobertura se harían los escaneos. Es interesante notar la ventaja de la cerrada en arco que permitiría realizar el escaneo desde una sola estación si ésta se pudiera colocar centrada. Por el contario en el caso de paramento recto los ángulos de incidencia en los extremos son más agudos por lo que probablemente sería necesario estacionar el TLS en más de una posición. Las visuales desde las laderas del cauce aguas abajo obligarían a estacionar varias veces, principalmente por motivo de distancia ya que en el caso de cierre en arco la incidencia angular sigue siendo buena.

Tabla 4.Valores de exactitud en las medidas del ensayo.

\begin{tabular}{|c|c|c|c|c|}
\hline Punto & Medida & Distancia (m) & Ángulo (g) & Exactitud (mm) \\
\hline $\mathbf{1}$ & B4-Zona1 & 88,5 & 92,3 & 2,6 \\
\hline $\mathbf{2}$ & B5-Zona1 & 105,1 & 80,4 & 2,6 \\
\hline $\mathbf{3}$ & B4-Zona2 & 109,2 & 59,6 & 2,7 \\
\hline $\mathbf{4}$ & B5-Zona2 & 132,3 & 54,7 & 3,4 \\
\hline
\end{tabular}



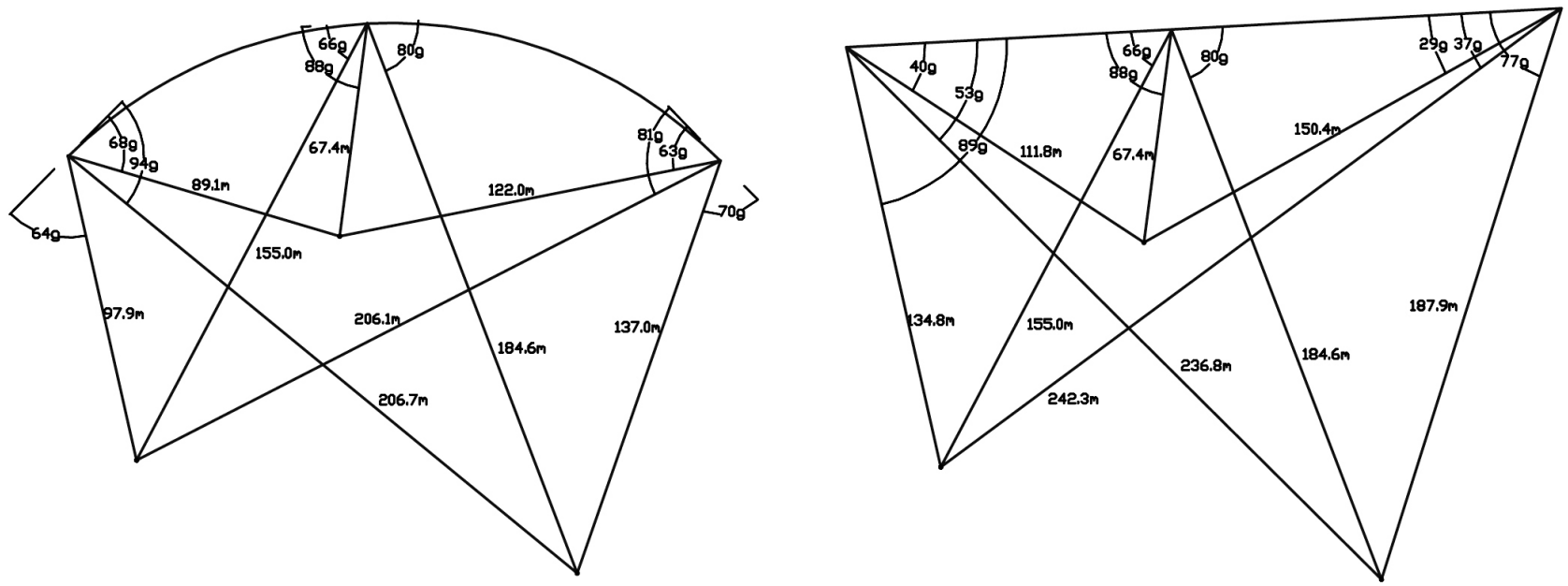

Figura 8. Comparación de distancias y ángulos de incidencia entre una presa en arco y una presa recta.

En la mayoría de las situaciones nos veríamos obligados a realizar observaciones desde estaciones diferentes, lo que nos llevará a la necesidad de realizar el registro de las diferentes nubes de puntos obtenidas. Esto plantea la necesidad de colocar y observar dianas para el correcto registro de las nubes de puntos.

Como se trata de obtener movimientos estacionales de la presa convendría colocar las dianas en puntos fijos del terreno. Se podrían aprovechar los vértices de la red local donde también se hace la puesta en estación del TLS y se podría además colocar algún punto fijo en los estribos. La mayor operatividad se consigue si se dispone de tantas dianas como puntos fijos y se colocan todas inicialmente. Luego se procede a estacionar el TLS en los vértices elegidos retirando la diana del vértice que corresponda y volviendo a colocar la diana una vez terminados las escaneos desde dicho vértice. Desde cada estación del TLS se deben tomar medidas de las otras dianas que estén a una distancia inferior a los límites del aparato de acuerdo con una gráfica equivalente a la de la Figura 2. Es por tanto necesario que desde cada estación del TLS se puedan observar al menos dos dianas.

\subsection{Consideración de la aplicación de los gráficos patrón dispersión/precisión y exactitud para otras estructuras}

En el seguimiento de movimientos de estructuras, se ha aplicado el TLS en edificios altos (11), con problemas en la cimentación (12) y en puentes (13). Todo lo expuesto sería aplicable a estos nuevos casos. El caso de las presas tiene una ventaja ya comentada, la preexistencia de una red local georreferenciada; esto es fundamental para una economía de medios y reducción de tiempos de toma de datos y post-proceso. No obstante, si se tratase de un trabajo periódico, es recomendable siempre que sea posible establecer una red local de puntos fijos donde colocar el TLS y las dianas.

\section{CONCLUSIONES}

Los equipos de medida TLS representan la punta de lanza en la transformación que se está produciendo en la Topografía terrestre, que se integra en la Geomática, pasando de las medidas puntuales a las superficies. El producto del TLS, la nube de puntos y la malla generada a partir de ésta, dan una visión global del objeto observado, permitiendo modelizar el mismo e incorporar nuevos tipos de análisis.

La principal ventaja de esta técnica es que permite obtener modelos continuos de paramentos, fachadas y estructuras de una manera rápida y segura en campo. Entre los inconvenientes principales se encuentran el tiempo de posproceso y la especialización requerida en la actualidad.

El grado de detalle que los fabricantes dan en las especificaciones de estos equipos, es insuficiente para planificar trabajos de ingeniería en espacios abiertos, por lo que se recomienda una metodología para analizar las prestaciones de estos equipos en un caso real y planificar estos trabajos de manera óptima.

Debido a su influencia en la precisión final, es importante conocer el alcance y nivel de incertidumbre de los distintos modelos de dianas empleados. Como conclusión de este estudio, se puede señalar que la diana esférica sirve para un rango de distancias mayor, y que, por su simetría espacial, no necesita orientarse hacia el TLS, como en el caso de las dianas planas de disco, más empleadas en trabajos a distancias cortas.

Los factores más determinantes en la precisión y exactitud de las medidas de los TLS son la distancia y el ángulo de escaneo. Frente al estudio individual de estos dos factores, se propone el estudio conjunto de ambos y la producción de gráficos patrón dispersión/precisión y exactitud para cada equipo, como documento de referencia para preparar una campaña de escaneos. Estos gráficos patrón deberían generarse para cada tipo de material y color.

Al preparar una campaña de toma de datos en campo, debe hacerse desde un planteamiento de economía de recursos y minimización del tiempo en campo. Siempre será preferible colocar más vértices en la red local que plantearse la colocación de dianas en puntos del paramento a observar, ya que no son fijos (más dianas) y de difícil acceso. Una buena estrategia es colocar dianas en todos los vértices de la red local, siempre que haya un número suficiente de vértices.

Las precisiones y exactitudes alcanzadas permiten realizar estudios de detalle de movimientos y deformaciones en es- 
tructuras y paramentos. Estos estudios son una fuente de datos para la calibración de los modelos 3D de deformaciones, lo que abre un nuevo escenario para la modelización de estructuras.

La precisión con la que el TLS detecta el detalle de los testigos puede ser también empleada como referencia del máximo detalle que podría ser detectado en el escaneo de un paramento, fachada, revestimiento o detalle constructivo. Por ello, la metodología propuesta en el presente artículo puede ser aplicada a otros campos de la ingeniería y arquitectura.

\section{AGRADECIMIENTOS}

A Rodrigo García, Jesús Bonet y Ángel Herranz de Leica Geosystems por el apoyo tecnológico, trabajo de campo y proceso de datos en el estudio de caso.

Este documento constituye un avance de la línea de investigación realizada en el Laboratorio de Topografía y Geomática de la E.T.S. de Ingenieros de Caminos, Canales y Puertos de la Universidad Politécnica de Madrid sobre Láser Escáner aplicado a la ingeniería civil y la construcción. Gracias a todos los compañeros del Laboratorio.

\section{REFERENCIAS}

(1) Pfeifer, N., Dorninger, P., Haring, A., Hongchao, F. (2008). Investigating terrestrial laser scanning intensity data: quality and functional relations. Optical 3-D Measurement Techniques VIII, 1(813): 328-337.

(2) Schulz, T. (2007). Calibration of a Terrestrial Laser Scanner for engineering geodesy. DISS. ETH No. 17036. Zurich.

(3) Mechelke, K., Kersten, T.P., Lindstaedt, M. (2007). Comparative investigations into the accuracy behaviour of the new generation of Terrestrial Laser Scanning systems. Optical 3-D Measurement Techniques VIII, 1(813): 319-327.

(4) European Leonardo Da Vinci Programme. (2008). 3DRiskMapping. Theory and practice on Terrestrial Laser Scanning. Training material based on practical applications. Bélgica: Flemish Agency of the European Leonardo Da Vinci programme.

(5) González-Aguilera, D., Gómez-Lahoz, J., Sánchez, J. (2008). A new approach for structural monitoring of large dams with a three-dimensional laser scanner. Sensors, 8(9): 5866-5883, doi: http://dx.doi.org/10.3390/s8095866.

(6) Marchamalo, M., Galán, D, Sánchez Sobrino, J.A., Martínez Marín, R. (2011). La tecnología DGPS en la construcción: control de movimientos en grandes estructuras. Informes de la Construcción, 63(522): 93-102, doi: http://dx.doi. org/10.3989/ic.10.0o8.

(7) Leica-Geosystems. http://www.leica-geosystems.es/downloads123/hds/hds/ScanStation\%20C10/brochures-datasheet/Leica_ScanStation_C10_DS_es.pdf.

(8) Rüeger, M. (2006). Overview of geodetic deformation measurements of dams. En ANCOLD 2006 Conference. Sydney, Australia.

(9) Galán, D, Martínez-Marín, R., Marchamalo, M., Sánchez-Sobrino, J.A. (2011). Control de movimientos en presas mediante DGPS. Aplicación a la presa de La Aceña (España). Tecnología y Ciencias del Agua, 2(3): 159-176.

(10) Alba, M., Bernardini, G., Giussani, A., Ricci, P. P., Roncoroni, F., Scaioni, M., Valgoi, P., Zhang, K. (2008). Measurement of dam deformations by terrestrial interferometric techniques. The International Archives of the Photogrammetry, Remote Sensing and Spatial Information Sciences, 37 Part B1(1374): 133-139. Beijing.

(11) Schneider, M. (2006). Terrestrial laser scanning for area based deformation analysis of towers and water dams. Proceedings of 3rd IAG Symposium of Geodesy for Geotechnical and Structural Engineering and 12th FIG Symposium on Deformation Measurements. Baden, Austria.

(12) Sternberg, H. (2006). Deformation measurements at historical buildings with terrestrial laser scanners. The International Archives of the Photogrammetry, Remote Sensing and Spatial Information Sciences, vol. XXXVI Part 5: $303-308$.

(13) Zogg, M. H., Ingensand, H. (2008). Terrestrial Laser Scanning for deformation monitoring-load test on the Felsenau viaduct $(\mathrm{CH})$. The International Archives of the Photogrammetry, Remote Sensing and Spatial Information Sciences, vol. XXXVII Part B5: 555-561. 